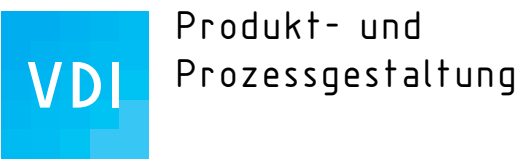

VDI-Berichte 2322

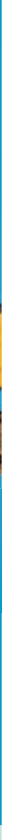

\title{
7. VDI-Tagung
}

Humanschwingungen 2018 
https://doi.org/10.51202/9783181023228-1

Generiert durch IP '172.22.53.54', am 26.04.2023, 13:25:48. 
VDI-BERICHTE

Herausgeber: VDI Wissensforum GmbH 
https://doi.org/10.51202/9783181023228-1

Generiert durch IP '172.22.53.54', am 26.04.2023, 13:25:48. 
Produkt- und

VDI Prozessgestaltung

7. VDI-Tagung

Humanschwingungen 2018

Würzburg, 24.-25. April 2018

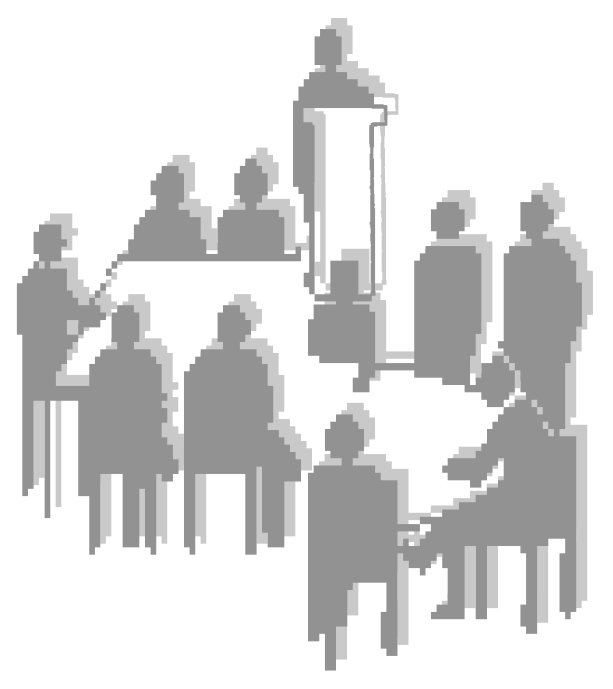

VDI-Berichte 2322 


\section{Bibliographische Information der Deutschen Bibliothek}

Die Deutsche Bibliothek verzeichnet diese Publikation in der Deutschen

Nationalbibliographie; detaillierte bibliographische Daten sind im Internet unter www.dnb.de abrufbar.

\section{Bibliographic information published by the Deutsche Bibliothek}

(German National Library)

The Deutsche Bibliothek lists this publication in the Deutsche Nationalbibliographie

(German National Bibliography); detailed bibliographic data is available via Internet at www.dnb.de.

\section{(C) VDI Verlag GmbH · Düsseldorf 2018}

Alle Rechte vorbehalten, auch das des Nachdruckes, der Wiedergabe (Photokopie, Mikrokopie), der Speicherung in Datenverarbeitungsanlagen und der Übersetzung, auszugsweise oder vollständig.

Der VDI-Bericht, der die Vorträge der Tagung enthält, erscheint als nichtredigierter Manuskriptdruck. Die einzelnen Beiträge geben die auf persönlichen Erkenntnissen beruhenden Ansichten und Erfahrungen der jeweiligen Vortragenden bzw. Autoren wieder.

Printed in Germany.

ISSN 0083-5560

ISBN 978-3-18-092322-2 
Inhalt

Seite

Vorwort

Einführungsvorträge

D. Wittekind, Schiffsschwingungen - Ein Überblick

P. Schnabel

R. Happee

Motion Comfort of Automated Driving a Biomechanical Modelling Approach

\section{Arbeitsschutz}

M. Köpfli Wicki Wie viele Arbeitnehmer sind in der Schweiz vibrationsgefährdet?

U. Nigmann

Hand-Arm-Vibrationen in Gießereien - Anforderungen,

Problemstellungen, Gefährdungsbeurteilungen

U. Schober, $\quad$ Fachbericht DIN SPEC 45674 über Kenntnisse zur

U. Kaulbars

Durchführung und Bewertung vonHumanschwingungsmessungen

\section{Hand-Arm-Schwingungen}

U. Kaulbars Messunsicherheit von Arbeitsplatzmessungen -

Orientierungswert für Hand-Arm-Vibration aus

Ringversuch

F. Pöhler, Komfortbewertung von Griffstellenvibrationen -

S. Hanußek

Entwicklung des „Komfortindex“ zur objektiven

Bewertung von Einzelstoßereignissen

S. Matthiesen,

Anforderungen an ein Messsystem zur Ermittlung der

A. Lindenmann, Rotationsimpedanz von Hand-Arm Systemen

T. Bruchmüller 


\section{Gesamtkörpervibrationen in Fahrzeugen}

C. Freitag,

Vibrationsexposition bei Aufsitzrasenmähern -

D. Sayn Evaluation des Schwingungsmessverfahrens nach DIN EN ISO 5395-1:2014

T. Strehlow,

Kundenoptimale Querführung beim teilautomatisierten

H. Oschlies, Fahren

F. Küçükay,

R. Henze,

S. Schmidt

A. Schwendicke, Zeitliche Maskierungseffekte von vertikalen Human-

J. Dou, schwingungen - Kurzer Vergleich von Vor- und Nach-

M. E. Altinsoy maskierung mit einem detaillierteren Blick auf Nachmaskierung

\section{Hand-Arm-Schwingungen}

G. Fleury

T. Schenk
Neue Prüfmethode zur Klassifizierung von Handhämmern nach ihrer Schwingungsübertragung

Ein Vorschlag für die Definition von diskreten Stößen, die auf das Hand-Arm-System des Menschen bei der Arbeit mit mechanisierten und nicht mechanisierten Werkzeugen einwirken 


\section{Gesamtkörpervibrationen in Fahrzeugen}
K. Zerle,
Bewertung des Schwingungsdosiswertes und Fahr-
K. Sepahvand, komfort im PKW unter Unsicherheiten
S. Marburg

M. Kremb, Relevanz zusätzlicher Bewertungsverfahren nach 189

C. von Holst, ISO 2631-1 bei der Beurteilung der Schwingungs-

C. Oberhaus exposition in Traktoren

\section{Experimentelle \& numerische Verfahren}

\section{A. Siefert,}

J. Hofmann

J. Keiner,

L. Vollmann

A. Veeraraghavan, Y. Lu,

J. Hofmann,
A. Siefert

R. Rosenkranz,

E. Altinsoy,

S. Gruschwitz,

D. Schecker

S. Lück,

R. Naumann,

M. Hermanski
Einbindung Aktiver Rumpfmuskulatur in ein FE Modell zur Analyse von Humanschwingungen

Dynamische Druckverteilungsmessungen zur Komfortbewertung

225

237 Sitz- und Ridekomfort

Nutzung von Wahrnehmungsmerkmalen für die 253 Beurteilung von Humanschwingungen - Wahrnehmungsmerkmale können als Grundlage für die systematische Beurteilung und Gestaltung von Ganzkörperschwingungen dienen

Experimentelle und simulationsgestützte Komfortunter265 suchungen an einem elektrischen Rollstuhl 
Seite

\section{Minderungsmaßnahmen}

C. Genau

K. Krivenkov,

H. Wittmann

J. Bokemeyer

O. Mönnich, H. Lehr,

J. Maas

\section{Poster}

D. Sayn,

C. Böser
Das neue PFERD-Werkzeugsystem als Alternative zu Schruppscheiben

Isolation von lateralen und longitudinalen Schwingungen

289 in gefederten Fahrersitzen von Nutzfahrzeugen:

Herausforderungen und Potentiale

Aktive Pneumatiklagerung für Fahrzeugsitze

299

Aktive Vibrationsminderung für einen handgeführten

309 